\title{
Tuberculosis: Laboratory Diagnosis and Dots Strategy Outcome in an Urban Setting: A Retrospective Study
}

\author{
Nazish Fatima ${ }^{*}$, Mohammad Shameem², Fatima Khan'1, Indu Shukla1, Parvez A. Khan1, \\ Nabeela ${ }^{1}$ \\ ${ }^{1}$ Department of Microbiology, Jawaharlal Nehru Medical College, Aligarh Muslim University, Aligarh, India \\ ${ }^{2}$ Department of TB \& Respiratory Diseases, Jawaharlal Nehru Medical College, Aligarh Muslim University, \\ Aligarh, India

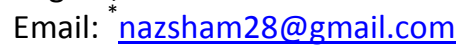

Received 23 May 2014; revised 26 June 2014; accepted 7 July 2014

Copyright (C) 2014 by authors and Scientific Research Publishing Inc.

This work is licensed under the Creative Commons Attribution International License (CC BY).

http://creativecommons.org/licenses/by/4.0/

(c) (i) Open Access

\section{Abstract}

Pulmonary Tuberculosis (TB) is a global public health threat. It has a worldwide distribution with a very high prevalence in Asian countries. A correct diagnosis forms an important and indispensable part of proper and timely treatment. Detection of acid fast bacilli in direct smears has considerable clinical and epidemiological value and remains the most widely used rapid diagnostic test. In smear negative cases, radiography has an important role to play. Directly Observed Treatment Short Course (DOTS) strategy is one of the most cost-effective health interventions to treat tuberculosis. Samples from clinically suspected cases of TB were screened for the presence of Acid Fast Bacilli (AFB). Radiography and culture was done for the AFB negative cases. 760 cases of pulmonary TB were diagnosed by smear examination and 412 by radiography. 270 cases of pulmonary TB were diagnosed in the study. Of the 1422 patients put on DOTS, out of which $1138(80.1 \%)$ were successfully treated. Thus, smear examination for AFB and radiography should go hand in hand for correct diagnosis and treatment of TB by DOTS which is a very successful regimen for developing countries like India.

\section{Keywords}

Tuberculosis, DOTS

\section{Introduction}

Pulmonary tuberculosis is a global public health threat. It has a worldwide distribution with a very high preva-

\footnotetext{
${ }^{*}$ Corresponding author.
}

How to cite this paper: Fatima, N., Shameem, M., Khan, F., Shukla, I., Khan, P.A. and Nabeela (2014) Tuberculosis: Laboratory Diagnosis and Dots Strategy Outcome in an Urban Setting: A Retrospective Study. Journal of Tuberculosis Research, 2, 106-110. http://dx.doi.org/10.4236/itr.2014.23013 
lence in Asian countries where $60 \%$ - $80 \%$ of children below the age of 14 years are affected. It is the single most leading cause of death due to an infectious agent. In India, there are approximately 500,000 deaths annually due to this disease. However, this trend can be reversed if the WHO recommended tuberculosis control strategy, the Directly Observed Treatment Short course (DOTS), is implemented. A full course of anti-TB drugs, sufficient to cure one patient, costs less than US $\$ 40$, making the DOTS strategy one of the most cost-effective health interventions. Therefore, the potential to significantly reduce tuberculosis epidemic already exists, provided continuous political and financial support is given to the programme [1].

A correct diagnosis forms an important and indispensable part of proper and timely treatment. Culture remains the gold standard for the definitive diagnosis of tuberculosis as it can detect cases with bacterial load of up to 10 - 100 bacilli/ml of sputum [2] and also allows testing for anti-tubercular drug susceptibility [3]. However, detection of acid fast bacilli (AFB) in direct smears has considerable clinical and epidemiological value and remains the most widely used rapid diagnostic test for tuberculosis in most developing countries [4]. Sputum smear negative cases present a challenge in tuberculosis management [5] as they are less infectious but are capable of transmitting the disease [6]. There have been reports that there are obvious lung lesions showing opacities and infiltration in patients whose sputum is negative by smear. X-ray radiography becomes important in such cases [7].

\section{Aims and Objectives}

This study was designed 1) to determine the case detection rate of pulmonary tuberculosis from the sputum samples; 2) to evaluate the role of radiography in the diagnosis of tuberculosis; 3) to evaluate the role of DOTS in the eradication of tuberculosis.

\section{Methodology}

The retrospective study was conducted at the DOTS centre, Department of TB \& Respiratory Diseases and the Department of Microbiology, Jawaharlal Nehru Medical College and Hospital, AMU Aligarh over a period of two years. A total of 3424 clinical samples from subjects suspected of tuberculosis were included in the study. Sputum smears were stained using Ziehl-Neelsen technique (ZN) and culture was performed on LowensteinJensen media and incubated at $37^{\circ} \mathrm{C}$ in $5 \% \mathrm{CO}_{2}$ for up to eight weeks for the detection of Mycobacterium. Smear negative patients were subjected to radiography. The X-ray chest was defined under three categories [1]: Extensive lesion with cavitation's, large nodular shadow and cavity [2]. Moderate lesion is consistent with tuberculosis, diffuse shadow and infiltration [3]. Lesion suspected other than tuberculosis. A patient was declared to have tuberculosis on chest X-ray if there was extensive lesion, moderate lesion or diffuse lesion and infiltration [8]. Patients diagnosed as cases of tuberculosis were treated under DOTS regimen. Follow up of the patients was carried out to assess treatment outcome. The outcome of the patient was divided on the basis of Revised National TB Control Programme (RNTCP) [9] definitions as:

- Relapse: A patient previously treated for TB, who has been declared cured or treatment completed, and is diagnosed to be bacteriologically positive (smear or culture) TB.

- Failure: A new smear positive patient who is smear positive at five months or later after starting treatment.

- Cured: A patient who is sputum-smear negative, in the last month of treatment and at least on one previous occasion.

- Died: A patient who dies for any reason during the course of treatment.

- Default: A patient whose treatment was interrupted for two consecutive months or more.

\section{Results}

A total of 3424 patients with clinical presentation of pulmonary and extra pulmonary tuberculosis were included in the study. Table 1 shows that fever with 3188 (93.1\%) cases and loss of appetite with 2753 (80.4\%) cases were the commonest symptoms at the time of presentation. Cough in 2205 (64.4\%), and weight loss in 1890 (55.2\%) cases were other important symptoms suggestive of pulmonary tuberculosis. Chest pain in 87 (2.5\%) cases, lymphadenopathy in 155 (4.5\%) cases, diarrhoea in 32 (0.9\%) cases, menstrual dysfunction in $28(0.8 \%)$ cases and neurological manifestations in $13(0.4 \%)$ cases were observed in patients of extra-pulmonary tuberculosis at the time of presentation. 
Table 1. Presenting complaints of patients suspected of tuberculosis.

\begin{tabular}{cc}
\hline Presenting complaints & No. (\%) \\
\hline Fever & 93.1 \\
Loss of appetite & 80.4 \\
Cough & 64.4 \\
Weight loss & 55.2 \\
Lymphadenopathy & $155(4.5)$ \\
Chest pain & $87(2.5)$ \\
Diarrhoea & $32(0.9)$ \\
Menstrual dysfunction & $28(0.8)$ \\
Neurological manifestations & $13(0.4)$ \\
\hline
\end{tabular}

All the 3424 patients were tested for acid fast bacilli in sputum smear stained by Z-N technique. Out of this, 760 (22.2\%) were positive for acid fast bacilli (Table 2 shows results of various diagnostic tests). 740 (97.4\%) of these were treated under DOTS and the remaining 20 (2.6\%) AFB positive patients were put on non-DOTS regimen. $412(15.5 \%)$ of smear negative patients were also put on DOTS based on the radiological findings suggestive of TB. Culture examination was done for all the smear negative patients suspected of tuberculosis on radiography and was found positive in $76(18.4 \%)$ of patients. Two hundred and seventy suspected cases of extra pulmonary tuberculosis were also put on DOTS. Lymph node TB (52.6\%) was the most common extrapulmonary TB followed by pleural (32.2\%), abdominal \& genital (8.1\%) and central nervous system (3.7\%) TB (Table 3).

Out of a total of 1422 patients put on DOTS, 1138 (80.1\%) were successfully treated and were declared cured. 81 (5.7\%) defaulted during the course of treatment, 63 (4.4\%) were cases of relapse, 99 (6.9\%) were declared failure cases while 41 (2.9\%) died before the completion of treatment (Figure 1).

\section{Discussion}

Tuberculosis is a global disease which has affected millions of people across the world. Due to the risk of spread of the disease and the potential for the emergence of drug resistant strains, accurate diagnosis and treatment of TB is very important.

According to the WHO guidelines for TB control, patients with more than two weeks history of cough should be screened for pulmonary TB with direct sputum smear examination for M. tuberculosis [10]. Because the signs and symptoms of TB are not specific, the fundamental principle for the diagnosis of TB is the accurate demonstration of $M$. tuberculosis in a suitable specimen from the suspected cases of pulmonary TB for the accurate treatment. Either diagnosis is based on the demonstration of tubercle bacilli in sputum or TB may be demonstrated in culture. In case of pulmonary TB, X-ray examinations have been used frequently as a primary screening method [11].

In the present study, a total of 3424 patients were suspected of TB based on the clinical presentation. Fever (93.1\%), loss of appetite (80.4\%), cough (64.4\%) and chest pain (32.2\%) were the main presenting features. 760 (21.2\%) of the clinically suspected cases were found positive for AFB by ZN staining. Microbiological examination by ZN smear is currently the most rapid method for the detection of M. tuberculosis but it has been reported to have a very low sensitivity of $33.8 \%$ [12]. Direct smear examination is relatively easy to perform, much less expensive than radiography or culture. The fact that the diagnosis of TB in persons discharging large amounts of bacilli may be established and treatment started on the same day is without doubt the greatest operational advantage of smear microscopy. It reduces to minimum "losses" of patients due to long waiting periods, and it is also the only diagnostic method practicable almost everywhere.

The real challenges are patients who are clinically suggestive of TB but are sputum smear negative. The rapid tool which can help in diagnosing such patients is X-ray chest [13]. Four hundred and twelve such AFB negative patients were found to be radiologically suggestive in our study and were put on DOTS. On sputum culture of all these smear negative, radiologically active patients, only $18.5 \%$ showed growth on LJ media. Although culture is considered the gold standard for the diagnosis of TB [7], it has a low sensitivity (48.9\%) [11] and takes around 6 weeks to confirm the diagnosis. 
Table 2. Distribution of positive cases according to different diagnostic methods.

\begin{tabular}{cccc}
\hline Method & $\begin{array}{c}\text { Positive cases } \\
\text { No. (\%) }\end{array}$ & $\begin{array}{c}\text { Negative } \\
\text { No. (\%) }\end{array}$ & Total \\
\hline ZN staining & $760(22.2)$ & $2664(77.8)$ & 3424 \\
Culture & $28(18.5)$ & $123(81.5)$ & 412 \\
X-ray & $412(15.5)$ & $2252(84.5)$ & 2664 \\
\hline
\end{tabular}

Table 3. Site distribution among extra-pulmonary cases of tuberculosis.

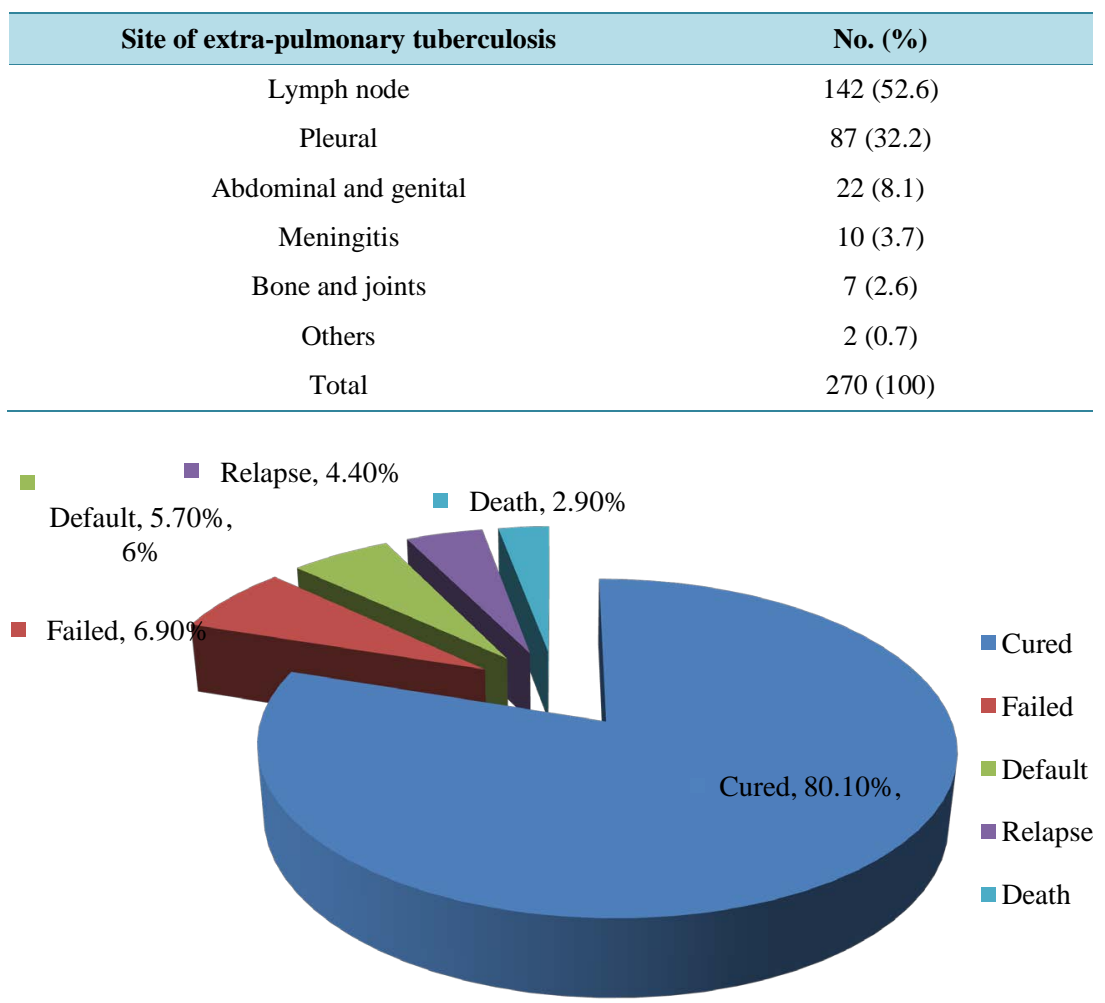

Figure 1. Treatment outcome of patients put on DOTS.

Smear and culture negative clinically suggestive cases having radiological opacities have been reported to respond to anti-TB drugs [13] [14]. Therefore, DOTS regimen was initiated in all the smear negative radiologically suggestive cases (412). Out of the 760 AFB positive patients, 740 were put on DOTS and 20 were put on non-DOTS regimen. 270 patients with extra-pulmonary TB were also put on DOTS. Cure rate at the end of the DOTS regimen was found to be $80.1 \%$ which is similar to what has been reported by other studies in India [15]. $81(5.7 \%)$ patients defaulted before the completion of treatment. The rate of defaulters is comparable to the RNTCP norm of 5\% [15]. Default could be because of false sense of cure due to early remission of symptoms or due to the poor drug compliance because of the adverse drug reactions of ATT like nausea, vomiting, skin rash etc.

The failure rate in our study was much higher from the RNTCP norm of $2 \%$. Nearly $7 \%$ cases were declared failed and $4.4 \%$ relapsed during the course of treatment. These could be the patients with MDR (multidrug resistant) or XDR (extensively drug resistant) strains of $M$. tuberculosis. Forty one (2.9\%) patients died during the treatment. The fatality rate is a little higher than the RNTCP norm $(<2 \%)$ but is lower than that reported in other parts of India (6.8\%) [15].

\section{Conclusion}

Acceptance of DOTS strategy in the Indian RNTCP has certainly brought encouraging success in the manage- 
ment of TB cases within the country. However, there are challenges to be met in the programme. In order to achieve effective TB control programme, effective guidelines, training and resources for good TB case management should be given a priority. An ideal programme ensures patient friendly services, accountability from health workers, continuous and accurate analysis of data for evaluation and self-sustained positive feedback.

\section{References}

[1] Blanc, L., Il Ahn, D. and Diletto, C. (2012) Guidelines for the Control of Tuberculosis. Through DOTS Strategies in Pacific Island Countries.

[2] Kamal, S.M.M., Mahmud, A.M., Ahsan, C.R., Islam, M.R., Sarwar, G., Rahman, M.M. and Barua, P.C. (2009) Bangladesh Journal of Medical Microbiology, 3, 23-26.

[3] Bello, A.K. and Njoku, C.H. (2005) Tuberculosis: Current Trends in Diagnosis and Treatment. Nigerian Journal of Clinical Practice, 8, 118-124.

[4] Truffot-Pernot, C., Veziris, N. and Sougakoff, M. (2006) Modern Diagnosis of Tuberculosis. La Presse Médicale, 35, 1739-1746. http://dx.doi.org/10.1016/S0755-4982(06)74892-8

[5] Siddiqui, K., Newell, J.N., Vander, S.P. and Gutezzo, E. (2006) Improving Sputum Microscopy Services for the Diagnosis of Tuberculosis in Peru and Bolivia. International Journal of Tuberculosis and Lung Disease, 11, 323-331.

[6] Behr, M.A., Warren, S.A., Salamon, H., Hopewell, P.C., Pondez, D., Leon, A. and Daley, C.L. (1999) Transmission of Tuberculosis from Smear Negative Patients: A Molecular Epidemiology Study. Lancet, 353, 444-449. http://dx.doi.org/10.1016/S0140-6736(98)03406-0

[7] Bah, B., Sow, V.M., Shiwanda, M., Camara, M.I., Larouze, B. and Murray, J.F. (2005) Useful Clues to the Presence of Smear-Negative Pulmonary Tuberculosis in a West African City. International Journal of Tuberculosis and Lung Disease, 6, 592-598.

[8] (2012) Revised National Tuberculosis Control Programme. DOTS-PLUS Guidelines. http://health.bih.nic.in/Docs/Guidelines-DOTS-Plus.pdf

[9] Shabbir, I., Iqbal, R. and Khan, S.U. (2007) An Analysis of Sputum Smear and X-Ray Results in Diagnosis of Smear Negative Pulmonary Tuberculosis. Pakistan Journal of Medical Research, 46, 67-69.

[10] World Health Organization (2004) Global Tuberculosis Control. WHO Report. WHO/CDSIB, Geneva.

[11] Suitters, B.T. and Brogger, S.A. (1967) Some Aspects of Laboratory Investigations in a Mass Campaign against Tuberculosis. Bulletin of the World Health Organization, 36, 837-845.

[12] Negi, S.S., Khan, S.F., Gupta, S., Pasha, S.T., Khare, S. and Lal, S. (2005) Comparison of the Conventional Diagnostic Modalities, Bactec Culture and Polymerase Chain Reaction Test for Diagnosis of Tuberculosis. Indian Journal of Medical Microbiology, 23, 29-33. http://dx.doi.org/10.4103/0255-0857.13869

[13] Sanchez, A., Gerhardt, G. and Naatal, S. (2005) The Need for Longitudinal Screening Studies in Prison TB Controls. International Journal of Tuberculosis and Lung Disease, 9, 633-639.

[14] Anthony, S., Douglas, S. and Gorden, L. (1989) Crofton \& Douglas—Respiratory Disease. 4th Edition, Delhi Oxford University Press, 395.

[15] Moharana, P.R., Satapathy, D.M., Sahani, N.C., Behera, T.R., Jena, D. and Tripathy, R.M. (2009) An Analysis of Treatment Outcome among TB Patients Put under DOTS at a Tertiary Level Health Facility of Orissa. Journal of Community Medicine, 5, 1-10. 
Scientific Research Publishing (SCIRP) is one of the largest Open Access journal publishers. It is currently publishing more than 200 open access, online, peer-reviewed journals covering a wide range of academic disciplines. SCIRP serves the worldwide academic communities and contributes to the progress and application of science with its publication.

Other selected journals from SCIRP are listed as below. Submit your manuscript to us via either submit@scirp.org or Online Submission Portal.
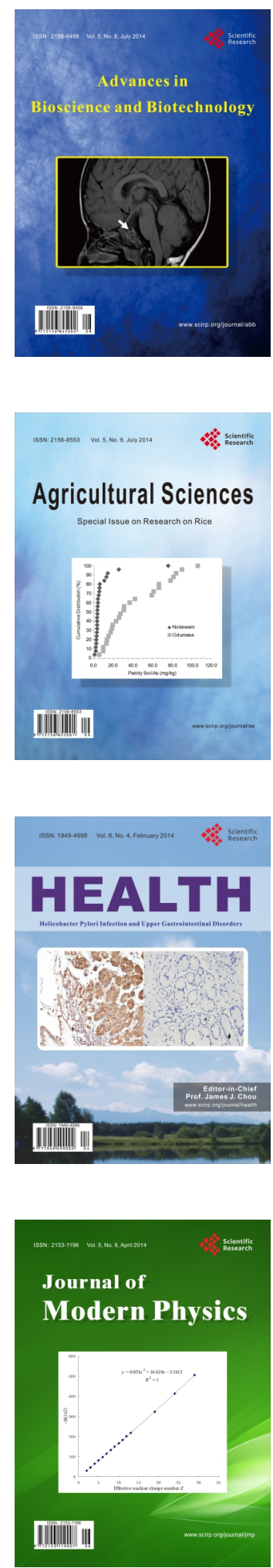
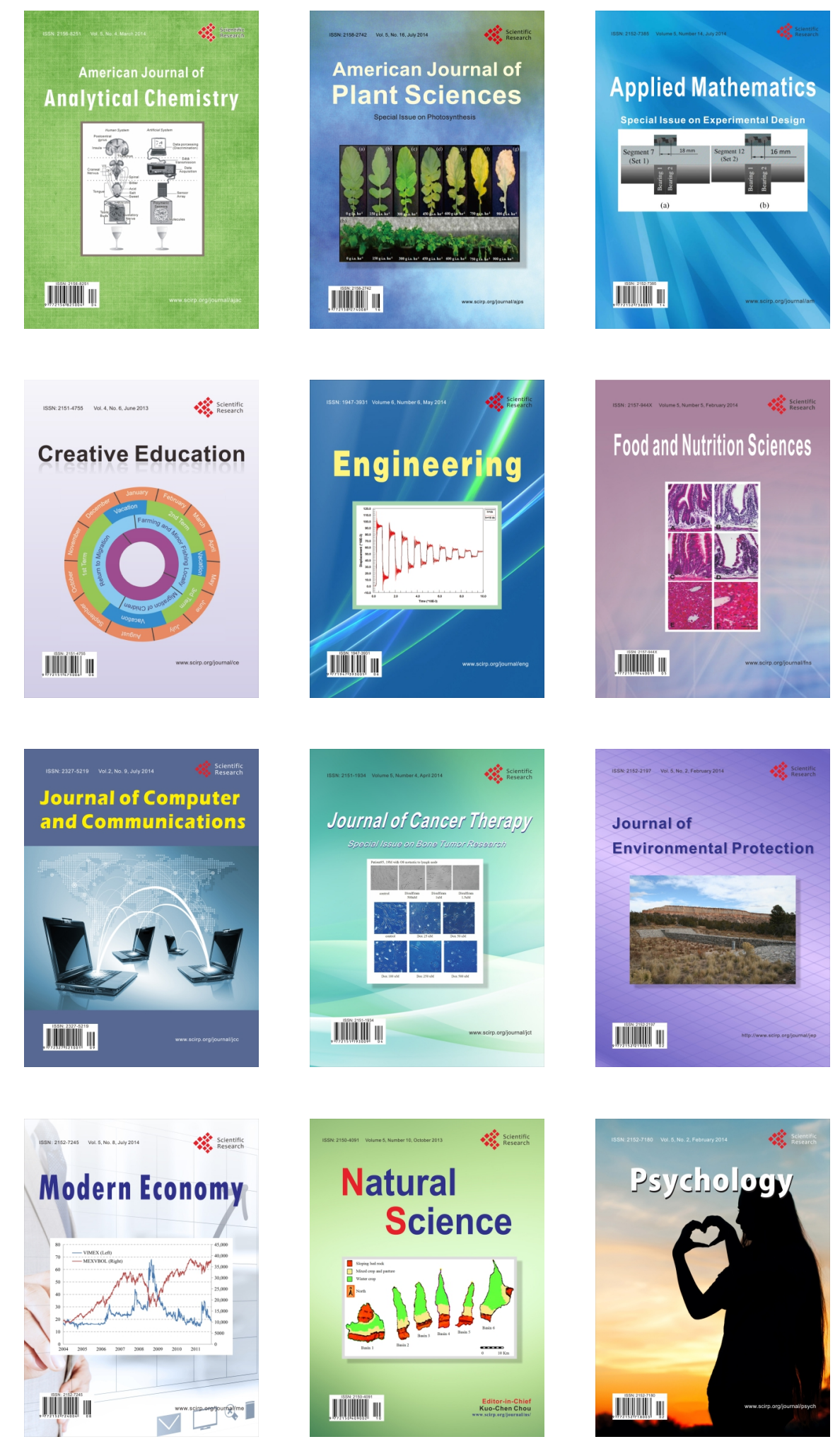